\title{
Strates
}

STRATES Matériaux pour la recherche en sciences sociales

8 | 1995

La question de l'environnement: recherches parallèles en Espagne et en France

\section{Le faste et le merveilleux, l'humilité et la mélancolie}

Deux discours fondateurs de la protection de la nature dans les deltas du Guadalquivir et du Rhône (1840-1910)

Bernard Picon

(2) OpenEdition

Journals

Édition électronique

URL : http://journals.openedition.org/strates/902

DOI : $10.4000 /$ strates.902

ISSN : $1777-5442$

Éditeur

Laboratoire Ladyss

Édition imprimée

Date de publication : 30 juin 1995

ISSN : 0768-8067

Référence électronique

Bernard Picon, "Le faste et le merveilleux, l'humilité et la mélancolie », Strates [En ligne], 8 | 1995, mis en ligne le 20 décembre 2005, consulté le 08 septembre 2020. URL : http://journals.openedition.org/ strates/902; DOI : https://doi.org/10.4000/strates.902

Ce document a été généré automatiquement le 8 septembre 2020.

Tous droits réservés 


\section{Le faste et le merveilleux, l'humilité et la mélancolie}

Deux discours fondateurs de la protection de la nature dans les deltas du Guadalquivir et du Rhône (1840-1910)

\section{Bernard Picon}

1 Deux grandes zones humides méditerranéennes, deux deltas importants, le delta du Guadalquivir et le delta du Rhône, situés pour l'un en Espagne, pour l'autre en France bénéficient aujourd'hui du statut d'espaces protégés.

2 En 1927 est créée dans sa partie centrale et sur 15000 hectares la Réserve Nationale de Camargue, en 1973 l'ensemble du delta, soit 75000 hectares, est érigé en Parc Naturel Régional.

3 En 1964 est créée la réserve biologique de Doñana au coeur du delta du Guadalquivir sur 6754 hectares. En 1969, 35000 hectares deviennent le Parc National de Doñana.

La naissance de la réserve de Camargue en 1927 : une exception

4 On peut déjà remarquer que la première opération de mise en réserve d'une zone humide est en France en avance de quarante ans sur l'Espagne. Cette différence temporelle ne traduit pas, en fait, une différence très profonde entre les deux pays sur l'émergence de l'idéologie de la protection de la nature.

5 Il a été démontré pour le cas français ${ }^{1}$ que la naissance de la Réserve Nationale de Camargue relevait de la solution à un conflit économique important autour de la gestion hydraulique du delta entre l'agriculture irriguée et l'industrie salinière. La zone centrale du delta constituait le lieu d'écoulage naturel et gravitaire des eaux douces de drainage de l'agriculture irriguée, mais était aussi utilisée par l'industrie salinière comme surface de concentration en sel. Cette incompatibilité de gestion hydraulique entre ceux qui adoucissaient ce milieu par des apports d'eau douce, et ceux qui cherchaient à le saliniser au maximum par apports d'eau de mer, se traduisit par la création de milieux saumâtres permanents dont la productivité biologique attira l'attention de quelques naturalistes de la Société nationale d'Acclimatation de France. 
6 En 1906, l'industrie salinière (Compagnie Alais Froges Camargue), propriétaire de cette zone conflictuelle constituée par l'étang du Vaccarès et des étangs périphériques, assigne les principaux propriétaires de Camargue devant le tribunal civil de Tarascon. Elle obtient gain de cause (confirmé par la cour d'appel d'Aix-en-Provence en 1907) et les agriculteurs sont interdits de tout rejet d'eau douce dans ce système Vaccarès, malgré le fait qu'ils fassent valoir qu'il s'agit d'un « droit coutumier ».

7 Les agriculteurs ne respecteront jamais les clauses de ce procès et continueront de déverser abondamment leurs eaux de drainage dans la propriété des salins. Les salins finiront par renoncer à l'exploitation de ces 15000 hectares trop adoucis par les agriculteurs. Ils se mettront d'accord avec ceux-ci pour confier en 1927 la gestion de la zone conflictuelle à la Société nationale d'Acclimatation de France qui avait mis en évidence l'originalité et l'intérêt scientifique de la productivité biologique de cet espace lacustre.

8 Cette première mesure de protection n'aurait pu voir le jour dès cette époque si elle n'avait pas pu permettre de trouver une solution à ce grave conflit. Il ne faut pas oublier en effet que l'idée protectionniste était à l'époque extrêmement minoritaire, surtout en ce qui concernait les milieux lacustres et les marais. Il est cependant remarquable de pouvoir faire la démonstration que ce qu'on appelle le sanctuaire de Nature de Camargue, la Réserve Nationale, est en fait une production "sociale » de nature en ce sens où les écosystèmes tirent leurs caractéristiques d'une artificialisation $\mathrm{du}$ fonctionnement hydraulique du delta. Par ailleurs, on peut constater que le point de départ de la protection de la nature est ici un déni de justice : les écosystèmes lacustres de la Réserve Nationale de Camargue sont "illégaux", en ce sens qu'ils doivent à l'origine leurs niveaux d'eaux et leurs taux de salinité aux apports d'eau agricole, considérés comme illégaux depuis 1907. L'idée de la protection de la nature sur un espace suffisamment vaste ne s'est donc pas imposée ici pour elle-même dès le départ. Elle a été une occasion à saisir, la justification, mais surtout la solution la plus pratique pour régler un conflit de gestion territoriale entre les agriculteurs du nord de la Camargue et l'industrie salinière du sud qui ont trouvé judicieux de se doter d'un no man's land, d'une zone tampon pour s'éviter de futurs conflits.

9 La leçon que l'on peut tirer de l'événement sur le plan sociologique est que des interactions sociales localisées apparemment anecdotiques contribuent pour une part non négligeable au changement social ou culturel, représenté en l'occurrence ici par l'apparition première de ce qui deviendra ultérieurement une évidence culturelle et une représentation sociale dominante : « la Camargue est un espace naturel ».

Les idées protectionnistes des "savants» de la Société nationale d'Acclimatation de France n'auraient pas pu trouver un ancrage social dans une Camargue où dominait à l'époque l'idéologie de la mise en valeur et de l'assèchement, si elles ne s'étaient révélées être le seul moyen de dénouer les fils d'un inextricable conflit local autour de la gestion de l'eau.

11 Il n'y avait vraisemblablement pas à l'époque dans le delta du Guadalquivir des conditions économiques locales qui auraient permis la mise en place d'une réserve nationale, pourtant le mouvement idéologique et culturel autour de l'idée protectionniste était notablement aussi présent en Espagne qu'en France. Ce n'est pas à cause d'un décalage dans le mouvement des idées entre la France et l'Espagne que l'on peut observer des décalages dans les législations sur la protection de la nature, mais ce sont plutôt des conditions locales particulières qui ont autorisé ou bloqué, dans un pays 
comme dans l'autre, la possibilité d'une concrétisation institutionnelle et territoriale de l'idée protectionniste.

Deux deltas, deux histoires parallèles

12 On se propose de démontrer, pour les deux espaces, la concomitance de l'émergence des discours tendant à valoriser ces territoires lacustres comme paysages exceptionnels, dignes d'être sauvegardés. Cette concomitance des discours n'induit pas pour autant un mimétisme du contenu de ceux-ci. Une fois encore, les spécificités locales vont contribuer à différencier les écrivains tentés par la défense et l'illustration de ces milieux et à intervenir sur l'argumentaire par eux utilisé.

Les cheminements symboliques qui aboutiront à la création du Parc National de Doñana en 1969 et à la création du Parc Naturel Régional de Camargue en 1973, s'ils sont au fond de même nature, n'ont cependant pas emprunté les mêmes voies. Ces différents itinéraires ont été dictés par les singularités locales et nationales. Il n'est pas question de revenir ici sur les histoires particulières des deltas du Rhône et du Guadalquivir, amplement développées dans deux ouvrages dont le propos était de mettre en évidence les mécanismes idéologiques, sociaux, économiques qui, dans leur confrontation historique avec les données naturelles de ces deltas, ont contribué à en faire les symboles de nature qu'ils sont devenus aujourd'hui ${ }^{2}$.

14 Ces deux approches ont par la suite donné lieu à une analyse comparative de la part des deux auteurs qui, après avoir évoqué ce qui différenciait les destins des deux deltas, ont focalisé leur analyse sur un certain nombre de convergences ${ }^{3}$. Ils ont ainsi d'abord mis en évidence comment les spécificités de ces terrains les ont préservés des investissements agricoles classiques des périodes anciennes. Puis ils ont montré comment ces milieux, considérés et utilisés comme zones privilégiées de prélèvement de ressources naturelles, ont constitué des enjeux importants entre les collectivités locales et les classes dominantes d'origine urbaine (aristocratie, bourgeoisie). Comment ensuite a émergé l'idée romantique du paysage exceptionnel, l'idée scientifique de l'exceptionnelle productivité biologique de ces milieux. Comment, en même temps, au nom du progrès, de l'hygiène, de la salubrité publique, de la productivité, sont apparus les grands projets de mise en valeur, représentations en contradiction avec les précédentes. Comment, à la suite de la réalisation de ces projets rendus possibles par les avancées technologiques, ces territoires artificialisés se sont partagés en zones productives et zones protégées, parfois complémentaires les unes des autres, notamment sur le plan de la gestion hydraulique ou sur le plan de la variété des milieux exploités par la faune. Comment finalement, sous-tendu par le discours scientifique et intériorisé par les classes moyennes urbanisées, c'est la tendance protectionniste qui l'emporte en tant que discours dominant. Ce discours dominant et les pratiques qu'il engendre restent conflictuels avec les discours et les pratiques des populations locales qui, confrontées à la précarité économique, continuent de vivre l'appropriation au nom de la protection de la nature comme quelque chose d'aussi illégitime que l'appropriation aristocratique des siècles passés.

L'émergence d'une nouvelle représentation façonnée par les singularités locales

Cette partie a pour ambition de s'en tenir au thème de l'émergence de cette nouvelle représentation des milieux lacustres comme paysages exceptionnels, contemporaine il est vrai de la perception de ceux qui voulaient les transformer en riches plaines agricoles à partir de 1840. Ce nouveau regard sur la nature est certainement à l'origine de l'institutionnalisation ultérieure des opérations de protection. Cette sphère des 
représentations, des idéologies, des valeurs, de la symbolique, est pour nous première parce qu'elle renvoie à des usages et des pratiques multiples qui, agrégées sous forme de mouvements sociaux, débouchent sur des règles et des normes qui s'institutionnalisent sous la forme de Parcs ou de Réserves.

16 L'hypothèse consiste à prétendre que les représentations nouvelles qui pouvaient émerger à la fin du siècle dernier sur les deux deltas en question étaient produites de manière générale par une nébuleuse intellectuelle européenne incluant savants, érudits, poètes, mais qu'elles étaient en partie déterminées par les singularités locales. Ce sont ces singularités locales qui ont accéléré ou freiné l'institutionnalisation de la protection de la nature. On peut se référer ici à l'histoire de la création du Parc Naturel Régional de Camargue, freinée pendant six ans, de 1968 à 1973, par la résistance des propriétaires locaux hostiles à la mise en place de ce projet émanant des sphères politiques parisiennes. Les propriétaires des grandes exploitations de Camargue nées de la difficulté de la mise en valeur de ce milieu et de son faible rendement agricole voyaient d'un mauvais oeil le projet de Parc Naturel Régional qui, pensaient-ils, ne pouvait qu'entraver leur logique d'agriculteurs modernistes. Leur assise sociale et politique les autorisa à bloquer le projet du nouveau ministère de l'Environnement jusqu'à ce que le ministre leur garantisse la majorité des sièges au conseil d'administration. Habitués depuis des siècles à modeler ce territoire par endiguement et irrigation, ils entendaient en garder la maîtrise. Ils ont eu gain de cause en contrôlant la fondation du Parc Naturel Régional. La forme juridique qu'a pris l'institution habilitée à protéger la nature en Camargue a bel et bien été modelée par la spécificité du milieu social local. Il en est allé de même avec les discours et les auteurs qui ont initié dès la fin du siècle dernier le mouvement qui a abouti à la protection institutionnelle de ces milieux.

17 Le delta du Rhône et le delta du Guadalquivir ont en commun d'avoir vu converger sur eux et aux mêmes époques les discours de la valorisation naturaliste et les discours de la mise en valeur agronomique. Ces deux représentations opposées n'ont pas engendré, comme pourrait le laisser supposer l'actuelle institutionnalisation de la protection de la nature, un combat d'idées entre les ingénieurs et les naturalistes, dont ces derniers seraient sortis vainqueurs. Le résultat de cet affrontement est tout à fait paradoxal puisque les endiguements, les irrigations, les drainages, les mises en valeur agricoles et industrielles n'ont en rien bloqué, à la fin du siècle dernier et au début de ce siècle, l'émergence puis le développement de l'idée que ces deltas constituaient des sanctuaires naturels, ainsi que culturels. Cela peut s'expliquer en partie, mais l'explication n'est pas suffisante, parce que ces opérations de mise en valeur n'ont jamais, dans un cas comme dans l'autre, débouché sur une mise en culture de la totalité de ces territoires. Le delta du Guadalquivir a vu sa rive gauche presque totalement vouée à l'agriculture industrielle, mais pas sa rive droite. La Camargue a été soumise à une intense colonisation agricole sur les bourrelets alluviaux des anciens cours du Rhône ainsi que sur les cours actuels, mais les terres basses, les marais doux et salés ont échappé à cette colonisation, ils étaient complémentaires à l'agriculture.

18 Ce n'est donc pas dans les particularismes physiques et paysagers de ces deux deltas qu'il convient de chercher les causes de leur désignation comme espaces naturels, mais peut-être plutôt dans les particularités des discours tenus sur eux à partir de la deuxième moitié du XIX ${ }^{e}$ siècle.

Doñana, le faste et le merveilleux 
19 Juan Francisco Ojeda Rivera fait remarquer à propos du delta du Guadalquivir qu'en effet, à partir de 1840-1850, deux images et deux conceptions de la gestion de ce territoire sont aux prises :

20 "Dans la création de l'image de ces milieux sont intervenus deux types de pionniers s'intéressant pour les uns à leur conservation, pour les autres à leur développement. Les seconds, ingénieurs, agronomes, forestiers, géologues et techniciens en général, s'identifiant avec des plans productivistes tant agricoles que financiers. Les premiers, chasseurs, voyageurs romantiques, naturalistes, mariant une conception élitiste de la chasse avec un fort sentiment romantique de la nature sauvage."

21 En ce qui concerne ce second groupe, les conservateurs romantiques, il est à noter qu'ils sont voyageurs et chasseurs. Cette caractéristique sociale du groupe des pionniers dont l'ambition est de protéger la nature en basse Andalousie ne se retrouve pas vraiment en France à propos de la Camargue. Ces voyageurs-chasseurs, pour beaucoup d'origine anglo-saxonne, à l'image de Richard Ford (1846), sont fascinés par l'abondance d'espèces-gibiers.

22 "Doñana est un des lieux de divertissement les plus délicieux d'Andalousie: on y rencontre lapins, lièvres, perdrix, palombes et surtout en grande abondance cerfs et sangliers. Ce territoire est en partie loué à des utilisateurs espagnols qui accueillent à certaines périodes une multitude d'étrangers, surtout anglais, dont la chasse est le principal souci. " ${ }^{4}$

23 A cette fascination pour l'aspect giboyeux du site s'ajoute la fascination pour la traditionnelle chasse des rois de Doñana :

24 "Les visites fastueuses de rois et de personnages célèbres se succèdent ajoutant un lustre supplémentaire à l'auréole romantique de Doñana. A la présence de Goya au printemps de 1797, qui immortalise la lagune de Santa Olalla, cadre paysager d'une de ses "majas", succèdent les chasses auxquelles participent, en octobre 1863 Eugènie de Montijo, ou le Prince Rodolphe de Habsbourg héritier d'Autriche-Hongrie, au printemps 1874, ou Alphonse XII en 1882. Le lien qui s'était noué entre Doñana et l'aristocratie sera consacré dans le premier tiers du XX $\mathrm{X}^{e}$ siècle avec la présence continuelle d'Alphonse XIII qui viendra par seize fois chasser à Doñana. " ${ }^{5}$

De plus ces chasseurs sont aussi esthètes: ils apportent des fusils, mais aussi des pinceaux.

26 «Raphaël Sanchez, journaliste à la revue de Cadix décrit ainsi en 1840 les participants à une chasse à Doñana : "Deux belles femmes de la région, gracieuses et élégantes, trois Anglais (...) qui après avoir chassé l'ours blanc dans les régions boréales du Spitzberg, ont troqué leurs fourrures pour le pantalon de coutil et chassent le lynx (...), cinq Espagnols et un Italien, jeune artiste au regard volcanique et à l'allure sombre équipé d'un énorme fusil arabe et d'une belle collection de pinceaux.," " 6

27 A ces discours cynégétiques mondains et esthétisants se rajouteront des préoccupations plus scientifiques sur la biologie des milieux :

28 "La richesse cynégétique de Doñana est une des constantes qui apparaissent dans les descriptions continuelles du site depuis les chroniques d'Alphonse $X$ et le livre d'Alphonse XI; puis la préoccupation scientifique d'effectuer un recensement de sa faune apparait en 1887 avec l'établissement de la première liste des oiseaux qui l'on pouvait observer dans les parages de Santa Olalla. " ${ }^{7}$ 
29 Au delà, à la fin du siècle dernier et au début de ce siècle, les observateurs rajoutent à leurs descriptions un certain nombre de considérations sur les aspects humains de la région et sur la question agraire.

"Deux livres écrits conjointement par les anglais Chapman et Buck ("Wild Spain», 1893 et "Unexplored Spain", 1910), (entretenant pour l'un des relations commerciales avec la ville de Jerez et pour l'autre, vice-consul britannique dans la même ville) font définitivement connaître Doñana au niveau mondial. Au delà des classiques descriptions littéraires de la nature sauvage, des bandits, des gitans, etc., l'importance de la question agraire dans le sud de l'Espagne à la fin du XIXe siècle et au début du XXe ne passe pas inaperçue aux yeux de ces deux auteurs. Comme ils l'indiquent dans la préface de Wild Spain leur prétention de " décrire une terre peu connue du point de vue inédit du sportif-naturaliste» ne les empêche cependant pas de faire "quelques observations complémentaires sur l'agriculture. ${ }^{8}$

31 S'ils annoncent que "Doñana a été leur terrain de chasse favori à toutes les époques de l'année ", Juan F. Ojeda Rivera fait remarquer que, malgré cela, leur analyse de la faune et des écosystèmes a été menée avec une perfection difficilement égalable.

La recherche de solutions pour la préservation de cette région partira de cette époque. Depuis le 19 juin 1911 existait en Espagne une commission royale de tourisme dont l'une des fonctions était de "veiller à la conservation et à la mise en représentation de l'Espagne artistique, monumentale et pittoresque ». Sous la $\mathrm{II}^{e}$ République, des intellectuels suggérèrent à l'I.R.A. (Institut de la Réforme Agraire) de faire de Doñana une « réserve nationale de tourisme ». En 1931, un agronome, Alfonso Aramburu, propose de faire de Doñana une "finca de utilidad social». Les propriétaires refusent cette formule préconisée par le Front populaire. Par ailleurs les populations riveraines souhaitent un partage des terres à des fins agricoles ou pastorales. Ce n'est qu'en 1957 que renaît l'idée protectionniste. Julian Huxley, prix Nobel de biologie déclare que «la séculaire chasse des rois et des princes peut être transformée en première réserve biologique d'Europe ». Cette phrase d'Huxley résume parfaitement le cheminement idéologique exposé dans les pages qui précèdent. Les déclarations des « chasseurs-naturalistes » qui, comme A. Chapman, écrivaient en 1910 «ici le printemps est un éden ornithologique (...) pour nous, chasseurs, naturalistes et amoureux de la vie sauvage, Doñana ne représente pas moins qu'un paradis sur terre", peuvent être considérées comme les textes fondateurs de la protection de la nature à Doñana.

La Camargue, l'humilité et la mélancolie

33 En Camargue, le combat opposant les tenants de la mise en valeur agricole et les tenants de la préservation est tout à fait bien résumé par ce discours tenu en 1922 aux Saintes Maries de la Mer par le marquis de Baroncelli-Javon :

34 «En Camargue, il faut bien se le dire, deux ennemis acharnés sont aux prises: d'un coté, ce que l'on veut appeler le progrès avec son cortège de machines et de destructions, ses nivellements, ses défrichements, ses digues, ses soldats (les riches qui se sont emparés du sol; de l'autre la Nature, la terre vierge, mère des taureaux et des chevaux sauvages, des flamants, des mirages, des légendes, de la poésie avec ses soldats aussi (moins riches d'argent, mais plus riches de coeur et de mémoire), les poètes, les savants, les gardians, les pêcheurs, les Gitans. " ${ }^{9}$

Le marquis de Baroncelli, "lou Marques", le fondateur du mythe de la Camargue "naturelle», appartient au mouvement du félibrige qui entend entretenir la culture provençale et sa langue. Avec quelques autres poètes (d'Arbaud, Aubanel, etc.) ils 
désigneront la Camargue comme le symbole territorial de résistance à un monde extérieur perçu comme dangereux. textes qui à la fin du XIX ${ }^{e}$ siècle décrivent la nature camarguaise somme symbole d'humilité : les terrains salés, l'absence de rentabilité de la maigre végétation halophile qui lui est liée, les marais, les roselières, ont inspiré bien des textes mélancoliques aux romantiques d'alors.

"Si un mélange abominable

et le désordre universel

n'emportaient pas notre race

avec les races d'ailleurs ;

si la barbarie qui à la porte heurte

voilà plus de sept cent ans

passait enfin le large

et respectait nos enfants. " ${ }^{10}$

A l'inverse des esthètes qui visitent Doñana, ils ne s'appuieront pas sur le faste, sur le merveilleux ni même sur la notion de plaisir pour valoriser ce territoire. Ils quitteront au contraire leurs riches bastides provençales ou leurs palais d'Avignon pour s'installer dans de modestes demeures camarguaises d'où ils plaideront pour les authentiques valeurs engendrées par des rapports vrais à la Nature. Ils défendront les humbles, ils leur redonneront leur fierté en créant la « nation gardianne » et en dotant les cavaliers gardiens de troupeaux d'un uniforme : le costume de gardian.

Cette entreprise de défense culturelle puise vraisemblablement ses racines dans les "Ses grands horizons tristes, ses broussailles rabougries, sa monotonie silencieuse (...) et jusqu'à ses mirages, aussi fréquents peut-être que dans le désert qui, au dessus de cette désolation, font flotter de féeriques paysages, indécis comme un rêve et s'évanouissent comme une illusion " ${ }^{11}$.

Les défenseurs des humbles que se voulaient les poètes du félibrige ont trouvé là matière à transposer sur le plan social et culturel ce romantisme naturaliste.

"Des taureaux noirs, des chevaux blancs errent en toute liberté dans les steppes indécises de la Camargue, et semblent les seuls maîtres de cette plaine étrange, dont le silence solennel et les horizons lointains produisent une impression d'indéfinissable tristesse. ${ }^{12}$

Cette entreprise de naturalisation du social est flagrante dans La Bête du Vaccarès écrit en 1924 par Joseph d'Arbaud : l'humble gardian qui vit dans sa cabane de roseaux voit avec tristesse s'engloutir dans un marais la "bête du Vaccarès", symbole de la Camargue traditionnelle qui s'évanouit comme les mirages évoqués par de la Mothe. On est très loin de la mise en scène des fastueuses chasses royales et aristocratiques de Doñana.

Le résultat sera pourtant le même parce que, dans un cas comme dans l'autre, dans la richesse ou dans la pauvreté, dans le faste ou dans l'humilité, les hommes et la nature sont considérés comme un tout harmonieux mais menacé qu'il conviendra de protéger. Un Parc Naturel Régional comme celui de la Camargue se donne en effet pour mission autant de protéger la nature que les activités humaines qui en sont les garantes.

Conclusion 
51 S'il existe une caractéristique commune entre ceux qui, en Andalousie comme en Provence, à la fin du siècle dernier, ont été les pionniers de la protection de ces deux deltas, c'est le romantisme.

Par contre, ce qui les sépare, c'est pour l'Andalousie un romantisme qui s'est imposé de l'extérieur par la voie des grands bourgeois et naturalistes anglo-saxons très marqués par la symbolique de la chasse royale et aristocratique de Doñana remontant au $\mathrm{X}^{\mathrm{e}}$ siècle, dont il fallait arracher la richesse cynégétique, devenue pour les besoins de la cause richesse biologique, aux appétits des hommes d'affaire. Contrairement à la situation française, ici les chasseurs ont été les ferments de la protection. Leur culture romantique et savante en même temps a permis d'associer à leur souci de préservation d'espèces gibiers le souci de préservation d'une nature qu'ils ont érigée à la fois en monument de richesse biologique et en monument symbolique. L'orientalisme ambiant était à la fois propice à des digressions poétiques sur la rencontre de l'Afrique et de l'Europe, et à des développements scientifiques sur la diversité biologique due au mélange d'espèces en provenance des deux continents. Les fouilles de l'archéologue Schulten à la recherche d'une hypothétique civilisation Tartessa engloutie dans les marismas ajoutait une touche de mystère à l'ensemble.

En Provence, le romantisme qui s'exprima beaucoup à travers le mouvement félibre, est un romantisme militant. La nature, les paysages étranges et sans limites des marais, les mélanges confus de l'eau, de la terre et du ciel, l'évocation d'une terre de poésie et de mirages d'où l'homme est bien souvent absent, les horizons sans limite, symbolisent la « liberté ». Liberté menacée par le centralisme républicain qui, avec son cortège de progrès, et son entreprise de déstabilisation des cultures provinciales lamine une «Provence éternelle » jusque dans ses racines linguistiques. Le discours romantique est ici provençal, il n'est pas un produit importé. La faune et la flore ne sont appelées à la rescousse que pour leurs évocations symboliques, humilité mais aussi richesse d'une civilisation menacée. Les bandes d'oiseaux migrateurs qui s'effacent derrière les horizons marins ne sont pas décrits comme richesse biologique ou cynégétique, mais évoquent la fin du monde, fin d'une supposée grandeur provençale.

"Par moment, passe l'ombre d'un vol d'oiseaux qui, en pointe, là-haut, coupent l'air et s'en vont vers la mer immense. " ${ }^{13}$

Les activités touristiques et équestres, à partir de 1870 , ont pour mission d'affirmer de façon héroïque (la nation gardianne) que quelque part des hommes valeureux (les gardians) entretiennent la flamme d'un peuple libre qui entend rester attaché à une terre libre de toutes barrières, symbole de résistance des minorités laminées par le monde moderne. Nostalgie du passé et lutte contre l'extérieur ont trouvé leur symbole dans cette basse Camargue aux horizons dégagés des signes de la modernité.

En fin de compte, la protection de la nature dans le delta du Guadalquivir doit ses textes fondateurs à l'hédonisme des chasseurs-esthètes de la fin du XIX $\mathrm{XIècle}^{\mathrm{e}}$ la protection de la Camargue les doit pour une bonne part à la résistance romantique des poètes du félibrige. Deux traductions d'un même mouvement minoritaire qui s'institutionnaliseront quatre-vingt ans plus tard quand les naturalistes savants prendront le relais et quand l'idée de protection de la nature prendra des formes quasi universelles (WWF, UICN, etc.). 


\section{NOTES}

1. Picon Bernard (1988), L'Espace et le temps en Camargue, Arles, Actes Sud.

2. Cf. Ojeda Rivera Juan F. (1987), Organización del territorio en Doñana y su entorno próximo (Almonte), Siglos XVIII-XX, Madrid, Instituto Nacional para la conservación de la naturaleza, et Picon Bernard, op. cit.

3. Cette analyse conjointe a donné lieu à un article consigné dans la revue de l'Institut de Géographie de l'Université de Provence : Bernard Picon \& Juan F. Ojeda Rivera (1993), « De la nature « ressource » à la nature institutionnalisée dans les deltas du Rhône et du Guadalquivir ", Méditerranée, 3-4.

4. Ojeda Rivera, op. cit., p. 205.

5. Op. cit., p. 207.

6. Op. cit. , p. 206.

7. Op. cit. , p. 207.

8. Op. cit.

9. Marquis de Baroncelli-Javon, préface de La Camargue Gardianne d'Elly, 1938, in D. Le Fur (1994), Expériences de paysages en Camargue, Mémoire de DEA, EHESS, Paris.

10. D'Arbaud, Chanson des tridents, 1924.

11. A. de la Mothe, Le proscrit de Camargue, 1877.

12. Ch. Lentheric, La Grèce et l'Orient en Provence, 1878.

13. M. Jouveau, En Camargue, 1909.

\section{RÉSUMÉS}

Les mesures de protection des deltas du Rhône et du Guadalquivir dans les années 1960-70 peuvent être envisagées comme l'aboutissement d'un mouvement d'idées produit par le romantisme de la fin du XIX ${ }^{e}$ siècle. Ce romantisme a cependant emprunté des voies différentes, liées aux particularismes locaux. En Espagne il s'est appuyé sur l'image merveilleuse, entretenue par des chasseurs esthètes anglo-saxons, de territoires giboyeux qui abritaient depuis le Moyen Age les fastes des chasses royales. En France les poètes du mouvement félibrige ont au contraire vanté, sur un mode mélancolique, l'humilité et l'authenticité d'une culture et d'une nature locales menacées de mort par de riches affairistes extérieurs.

\footnotetext{
Splendour and supernatural, humility and melancholy. Two initial discourses on nature preservation in the Guadalquivir and Rhône deltas (1840-1910).

The preservation measures implemented in the Guadalquivir and Rhône deltas during the sixties and the seventies can be considered as the outcome of the XIXth century romantic ideas. But, due to local specificities, they were differently interpreted in each delta. In Spain, the Anglo-saxons both hunters and aesthetes used to report the fantastic element of territories where luxurious royal huntings were supposed to have taken place since the Middle Ages. In France, the provençal «felibrige " poets have praised the humility and authenticity of local nature and culture as opposed to the threatening projects of rich non local businessmen.
} 
Lo fasto y lo maravilloso, la humilidad y la melancolía. Dos discursos fundadores de la protección de la naturaleza en los deltas del Guadalquivir y del Ródano (1840-1910).

Los medios de protección de los deltas del Guadalquivir y del Ródano en los años 1960-70 pueden ser considerados como el desenlace de un movimiento de ideas producido por el romanticismo de finales del siglo XIX. Este romanticismo, sin embargo, ha seguido vías diferentes ligadas a las respectivas particularidades locales. En España se ha basado en la imagen maravillada, mantenida por cazadores estetas anglosajones, de territorios abundantes en caza en donde se llevaban a cabo desde la edad media los fastos de las cacerías reales. En Francia los poetas del movimiento Felibrigio han exaltado de manera melancólica la humildad y autencidad de una cultura y naturaleza locales amenazadas de muerte por ricos negociantes foráneos.

\section{INDEX}

Mots-clés : Environnement, Espagne, Représentations de la nature, Protection de la nature, Symbolique, Romantisme, Particularismes, Camargue, Delta du Guadalquivir

Keywords : France, environment, Spain, Protection of nature, Reprensentations of nature, Symbolism, Romanticism, Local specificities, Guadalquivir Delta

Palabras claves : medio ambiente, España, Francia, Representaciones de la naturaleza, Protección de la naturaleza, Simbólico, Romanticismo, Particularismos, Camarga, Delta del Guadalquivir

\section{AUTEUR}

\section{BERNARD PICON}

Sociologue, co-directeur du DESMID (Dynamique écologique et sociale des milieux deltaïques, CNRS, Arles). Les deltas constituent pour lui des sites privilégiés pour analyser les interactions Nature-Société. Il a notamment publié L'Espace et le temps en Camargue (Actes Sud, 1988). 